\title{
Distributed fiber sensing technology: Currents and challenges
}

\author{
Kazuo Hotate \\ Department of Electrical Engineering and Information Systems, The University of Tokyo, 7-3-1 Hongo, Bunkyo-ku, \\ Tokyo 113-8656, Japan. \\ Email: hotate@sagnac.t.u-tokyo.ac.jp \\ Recibido / Received: 29/09/2011. Aceptado / Accepted: 07/10/2011.
}

\begin{abstract}
:
In this paper, distributed fiber sensing technologies are explained, showing principles and applications. Time domain technologies have been developed, at first, as ways to analyze the distributed information along the fiber. For example, in distributed strain measurement through the nature of Brillouin scattering, basic systems showed limitation in spatial resolution of about $1 \mathrm{~m}$. However, novel phenomena and new systems have recently been proposed, and the resolution has been much improved. Optical correlation domain techniques, in which interference of continuous lightwaves is manipulated to obtain the distributed information, have already realized superior performances, such as $1.6 \mathrm{~mm}$ spatial resolution and $1 \mathrm{kHz}$ sampling rate, in the fiber Brillouin distributed strain sensing. Applications, such as aircraft health monitoring, have also been demonstrated. Recently, "Brillouin dynamic grating" in a polarization maintaining fiber has been studied, and created various novel applications, including simultaneous distributed measurement of strain and temperature. With the correlation domain technique, other schemes, such as multiplexing systems for long-length FBG distributed sensors, have also been developed.
\end{abstract}

Key words: Brillouin Scattering, Brillouin Dynamic Grating, Distributed and Multiplexed Sensing.

\section{REFERENCIAS Y ENLACES / REFERENCES AND LINKS}

[1]. T. Horiguchi, M. Tateda, "BOTDA - Nondestructive measurement of single-mode optical fiber attenuation characteristics using Brillouin interaction: Theory", J. Lightwave Technol. 7, 1170-1176 (1989).

[2]. T. Horiguchi, T. Kurashima, T. Tateda, "Technique to measure distributed strain in optical fibers", IEEE Photonic. Tech. L. 2, 352-354 (1990).

[3]. X. Bao, D. J. Webb, D. A. Jackson, "22-km distributed temperature sensor using Brillouin gain in an optical fiber", Opt. Lett. 18, 552-554 (1993).

[4]. M. Nikles, L. Thévenaz, P. A. Robert, "Brillouin gain spectrum characterization in single-mode optical fibers", J. Lightwave Technol. 15, 1842-1851 (1997).

[5]. M. N. Alahbabi, Y. T. Cho, T. P. Newson, "150-km-range distributed temperature sensor based on coherent detection of spontaneous Brillouin backscatter and in-line Raman amplification", J. Opt. Soc. Am. B 22, 1321-1324 (2005).

[6]. K. Hotate, T. Hasegawa, "Measurement of Brillouin gain spectrum distribution along an optical fiber using a correlation-based technique - Proposal, experiment and simulation", IEICE T. Electron. E83-C, 405-412 (2000).

[7]. Y. Mizuno, Z. He, K. Hotate, “One-end-access high-speed distributed strain measurement with 13-mm spatial resolution based on Brillouin optical correlation-domain reflectometry", IEEE Photon. Technol. L. 21, 474-476 (2009).

[8]. X. Bao, A. Brown, M. De Merchant, J. Smith, "Characterization of the Brillouin-loss spectrum of singlemode fibers by use of very short (<10-ns) pulses", Opt. Lett. 24, 510-512 (1999). 
[9]. C.-H. Li, K. Nishiguchi, M. Miyakate, A. Malcita, M. Yokoyama, K. Kishita, T. Mizutani, N. Takeda, "PPPBOTDA method to achieve $2 \mathrm{~cm}$ spatial resolution in Brillouin distributed measuring technique", IEICE Technical Report, OFT 2008-13 (2008).

[10]. W. Li, X. Bao, L. Chen, "Differential pulse-width pair BOTDA for high spatial resolution sensing”, Opt. Express 16, 21616-21625 (2008).

[11].S. M. Foaleng, M. Tur, J. C. Beugnot, L. Thévenaz, "High spatial and spectral resolution long-range sensing using Brillouin echoes", J. Lightwave Technol. 28, 2993-3003 (2010).

[12]. Y. Koyamada, Y. Sakairi, T. Takeuchi, S. Adachi, "Novel technique to improve spatial resolution in Brillouin optical time-domain reflectometry", IEEE Photonic. Tech. L. 19, 1910-1912 (2007).

[13].A. W. Brown, B. G. Colpitts, K. Brown, "Dark-pulse Brillouin optical time-domain sensor with 20-mm spatial resolution", J. Lightwave Technol. 25, 381-386 (2007).

[14]. M. A. Soto, G. Bolognini, F. D. Pasquale, L. Thévenaz, "Long-range Brillouin optical time-domain analysis sensor employing pulse coding techniques", Meas. Sci. Technol. 21, 094024 (2010).

[15].K.-Y. Song, S. Yang, "Simplified Brillouin optical time-domain sensor based on direct modulation of a laser diode", Opt. Express 18, $24012-24018$ (2010).

[16]. K. Hotate, K. Abe, K.-Y. Song, "Suppression of signal fluctuation in Brillouin optical correlation domain analysis system using polarization diversity scheme”, IEEE Photonic. Tech. L. 18, 2653-2655 (2006).

[17]. K. Hotate, H. Arai, K.-Y. Song, "Range-enlargement of simplified Brillouin optical correlation domain analysis based on a temporal gating scheme", SICE J. Control, Meas. Syst. Integr. 1, 271-274 (2008).

[18]. K.-Y. Song, Z. He, K. Hotate, "Distributed strain measurement with millimeter-order spatial resolution based on Brillouin optical correlation domain analysis", Opt. Lett. 31, 2526- 2528 (2006).

[19]. K.-Y. Song, K. Hotate, "Distributed fiber strain sensor with 1-kHz sampling rate based on Brillouin optical correlation domain analysis", IEEE Photonic. Tech. L. 19, 1928-1930 (2007).

[20]. K.-Y. Song, M. Kishi, Z. He, K. Hotate, "High-repetition-rate distributed Brillouin sensor based on optical correlation-domain analysis with differential frequency modulation", Opt. Lett. 36, 2062-2064 (2011).

[21]. S. S. L. Ong, H. Kumagai, H. Iwaki, K. Hotate, "Crack detection in concrete using a Brillouin optical correlation domain analysis based fiber optic distributed strain sensor", Proc. $16^{\text {th }}$ Intern. Conf. on Optical Fiber Sensors, Nara, no. We3-3 (2003).

[22].S. S. L. Ong, M. Imai, Y. Sako, Y. Miyamoto, S. Miura, K. Hotate, "Dynamic strain measurement and damage assessment of a building model using a Brillouin optical correlation domain analysis based

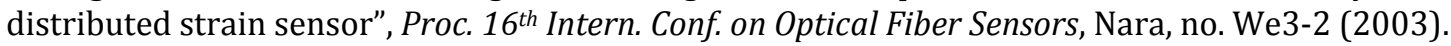

[23]. T. Yari, K. Nagai, M. Ishioka, K. Hotate, Y. Koshioka, "Aircraft structural health monitoring using onboard BOCDA system", Proc. SPIE 6933, 69330S (2008).

[24]. B. Zhu, T. Saida, K. Hotate, "Variable optical filter using dynamic grating in Er doped fiber controlled by synthesis of optical coherence function: Proposal and experimental verification", IEICE T. Electron. E86-C, 97-99 (2003).

[25].X. Fan, Z. He, K. Hotate, "Distributed strain sensor based on dynamic grating in polarizationmaintaining erbium-doped fiber", Opt. Lett. 33, 1647-1649 (2008).

[26].K.-Y. Song, K. Hotate, "All-optical dynamic grating generation based on Brillouin scattering in polarization maintaining fiber", Proc. 19 ${ }^{\text {th }}$ Intern. Conf. on Optical Fiber Sensors, Perth, no.3T-1, 7004219 (2008).

[27].K.-Y. Song, W. Zou, Z. He, K. Hotate, "All-optical dynamic grating generation based on Brillouin scattering in polarization-maintaining fiber", Opt. Lett. 33, 926-938 (2008).

[28]. W. Zou, Z. He, K.Y.-Song, K. Hotate, “Correlation-based distributed measurement of a dynamic grating spectrum generated in stimulated Brillouin scattering in a polarization-maintaining optical fiber", Opt. Lett. 34, 1126-1128 (2009).

[29]. K.-Y. Song, W. Zou, Z. He, K. Hotate, “Optical time-domain measurement of Brillouin dynamic grating spectrum in a polarization-maintaining fiber", Opt. Lett. 34, 1381-1383 (2009). 
[30].Y. Dong, X. Bao, L. Chen, “Distributed temperature sensing based on birefringence effect on transient Brillouin grating in a polarization-maintaining photonic crystal fiber", Opt. Lett. 34, 2590-2592 (2009).

[31].Y. Dong, L. Chen, X. Bao, "Truly distributed birefringence measurement of polarization-maintaining fibers based on transient Brillouin grating", Opt. Lett. 35, 193-195 (2010).

[32]. K.-Y. Song, H.-J. Yoon, "Observation of narrowband intrinsic spectra of Brillouin dynamic gratings", Opt. Lett. 35, 2958-2960 (2010).

[33] Y.-K. Dong L. Chen, X. Bao, "Characterization of the Brillouin grating spectra in a polarizationmaintaining fiber", Opt. Express 18, 18960-18966 (2010).

[34].V.P. Kalosha, W. Li, F. Wang, L. Chen, X. Bao, "Frequency-shifted light storage via stimulated Brillouin scattering in optical fibers", Opt. Lett. 33, 2848-2850 (2008).

[35].K.-Y. Song, K. Lee, S.-B. Lee, "Tunable optical delays based on Brillouin dynamic grating in optical fibers", Opt. Express 17, 10344-10349 (2009).

[36].W. Zou, Z. He, K. Hotate, "Tunable fiber-optic delay line based on stimulated Brillouin scattering", Appl. Phys. Express 3, 12501.1-3 (2010).

[37].S. Chin, N. Primerov, K.-Y. Song, L. Thévenaz, "True time reversal via dynamic Brillouin gratings in polarization maintaining fibers", Nonlinear Photonics Topical Meeting, no. NThA6, Karlsruhe, Germany (2010).

[38].W. Zou, Z. He, K. Hotate, "Complete discrimination of strain and temperature using Brillouin frequency shift and birefringence in a polarization-maintaining fiber", Opt. Express 17, 1248-1255 (2009).

[39].W. Zou, Z. He, K. Hotate, "Demonstration of Brillouin distributed discrimination of strain and temperature using a polarization-maintaining optical fiber", IEEE Photonic. Tech. L. 22, 526-528 (2010).

[40].W. Zou, Z. He, K. Hotate, "One-laser-based generation/detection of Brillouin dynamic grating and its application to distributed discrimination of strain and temperature", Opt. Express 19, 2363-2370 (2011).

[41]. R. K. Yamashita, W. Zou, Z. He, K. Hotate, "Measurement range elongation based on a temporal gating scheme in Brillouin correlation domain distributed discrimination system for strain and temperature operated by a single laser", Proc. $21^{\text {th }}$ Intern. Conf. on Optical Fiber Sensors (OFS-21), Ottawa, No. 7753-203 (2011).

[42]. K.-Y. Song, J.-H. Kim, H.-j. Yoon, "Simultaneous measurement of temperature and strain distribution by optical time-domain analysis of Brillouin dynamic grating" Proc. of $15^{\text {th }}$ OptoElectronics and Communications Conf. (OECC 2010), no. 9C4-4, Sapporo (2010).

[43].Y. Dong, L. Chen, X. Bao, "High-spatial-resolution time-domain simultaneous strain and temperature sensor using Brillouin scattering and birefringence in a polarization- maintaining fiber", IEEE Photonic. Tech. L. 22, 1364-1366 (2010).

[44]. M. Belal, T. P. Newson, "Enhanced performance of a temperature-compensated submeter spatial resolution distributed strain sensor", IEEE Photonic. Tech. L. 22, 1705-1707 (2010).

[45].K.-Y. Song, H.-J. Yoon, "High-resolution Brillouin optical time domain analysis based on Brillouin dynamic grating", Opt. Lett. 35, 52-54 (2010).

[46]. K.-Y. Song, S. Chin, N. Primerov, L. Thévenaz, "Time-domain distributed fiber sensor with $1 \mathrm{~cm}$ spatial resolution based on Brillouin dynamic grating", J. Lightwave Technol. 28, 2062-2067 (2010).

[47]. K. Hotate, K. Kajiwara, "Proposal and experimental verification of Bragg wavelength distribution measurement within a long-length FBG by synthesis of optical coherence function", Opt. Express 16, 7881-7887 (2008).

[48]. K. Kajiwara, K. Hotate, "Measurement of bragg-wavelength distribution in a long-length fiber Bragg grating with high speed sampling", Appl. Phys. Express 2, 082401 (2009).

[49]. K. Kajiwara, K. Hotate, "Multiplexed long-length fiber Bragg grating distributed sensors based on synthesis of optical coherence function", Proc. of $20^{\text {th }}$ Intern. Conf. on Optical Fiber Sensors, Edinburgh, OF101-84 (2009). 


\section{Introduction}

Optical fibers can sense strain, pressure and temperature through the optical properties, such as scattering, propagation-modes coupling, and so on. By applying appropriate ways to analyze distribution of these properties along the fiber, "fiber optic distributed sensing" can be realized. These sensor systems, which are called also as "fiber optic nerve systems," sense damages induced in materials and structures, such as aircraft wings, pipe lines, bridges and tunnels, in which the fiber is embedded. The materials and structures that can feel their pain are expected to be the key for enhancing the safety and security in the $21^{\text {st }}$ century society. In this paper, focusing on fiber Brillouin scattering as the sensing mechanism for temperature and/or strain, recent progress in the "fiber optic nerve systems" are reviewed, showing principles, performances, and applications. FBG based quasi-distributed sensing scheme is also discussed.

\section{Distributed sensing based on Brillouin scattering}

Brillouin scattering caused in an optical fiber has a frequency of about $11 \mathrm{GHz}$ down-shifted from the input lightwave. The shifted frequency is changed by temperature and/or longitudinal strain applied to the fiber. As a way to have the distributed information, a time domain technique with pulsed lightwave was proposed in 1989 [1,2]. This technique is classified into two schemes; one is "Brillouin Optical Time Domain Analysis (BOTDA)," in which stimulated Brillouin scattering caused by counterpropagating pump and prove lightwaves are used, and the other is "Brillouin Optical Time Domain Reflectometry (BOTDR)," in which the spontaneous scattering is detected by a heterodyne interferometer. Performance improvements had been demonstrated, and characterization of the measured Brillouin spectrum had also been performed [3-5]. On the other hand, our group proposed a technique named "Brillouin Optical Correlation Domain Analysis (BOCDA)" in 1999 [6]. It is based on control of correlation between the pump and probe continuous lightwaves, which excite the stimulated Brillouin scattering (SBS) at one selected position along the fiber. Recently, we also proposed "Brillouin Optical Correlation Domain Reflectrometry (BOCDR)" based on the spontaneous scattering [7].

In the basic systems for the time domain technique, the spatial resolution was limited around $1 \mathrm{~m}$, because of the spectrum broadening in the narrow optical pulse. However, some sophisticated schemes have recently been proposed to overcome the difficulty, utilizing pulse pre-pump [8,9], differential pulse-width pair [10], Brillouin echoes [11], double pulses [12], dark pulse [13], and so on. Spatial resolution in $\mathrm{cm}$ order has already been demonstrated. Other schemes in the time domain technique have also been proposed for enhancing the measurement range [14], or for simplifying the system [15].

In the correlation domain techniques $[6,16$ $19]$, it is the point that the pump and probe continuous lightwaves are identically frequencymodulated at the laser source. As a result, SBS occurs exclusively at the correlation peak position, where the two lightwaves are highly correlated [6]. The correlation peak width determines the spatial resolution. We can shift the peak position along the fiber by simply changing the FM frequency. Then, the distributed measurement can be achieved. Both in BOCDA and BOCDR, we can achieve random accessibility to arbitrary points to be measured. Measurement range of $1,020 \mathrm{~m}$ with the spatial resolution of $7 \mathrm{~cm}$ has been demonstrated [17]. The resolution of $1.6 \mathrm{~mm}$ has also been achieved, that is the highest resolution ever reported [18]. Sampling rate for one position has been improved to be $1 \mathrm{kHz}$ [19]. Recently, a way for automatically sweeping the correlation peak position has been proposed and demonstrated [20], as a collaborative work of our group and Chung-Ang University, Korea. With this technique, speed for the total distributed measurement by BOCDA has been much improved. Twenty traces of the total distributed measurement per one second have been demonstrated with $100 \mathrm{~m}$ range and $80 \mathrm{~cm}$ spatial resolution [20]. Applications of BOCDA have also been demonstrated, including sub-mm 


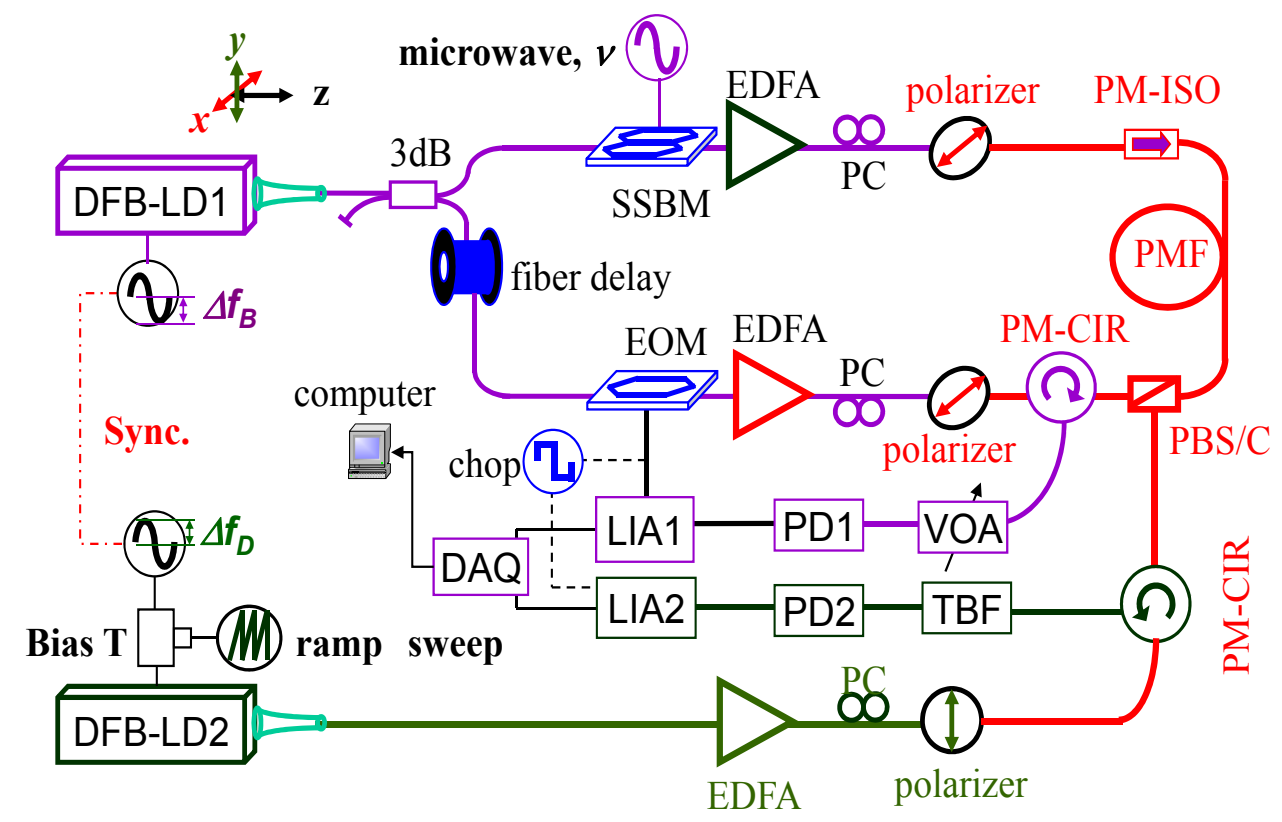

Fig. 1: System for distributed discriminative measurement of strain and temperature with Brillouin dynamic grating and correlation domain technique [39].

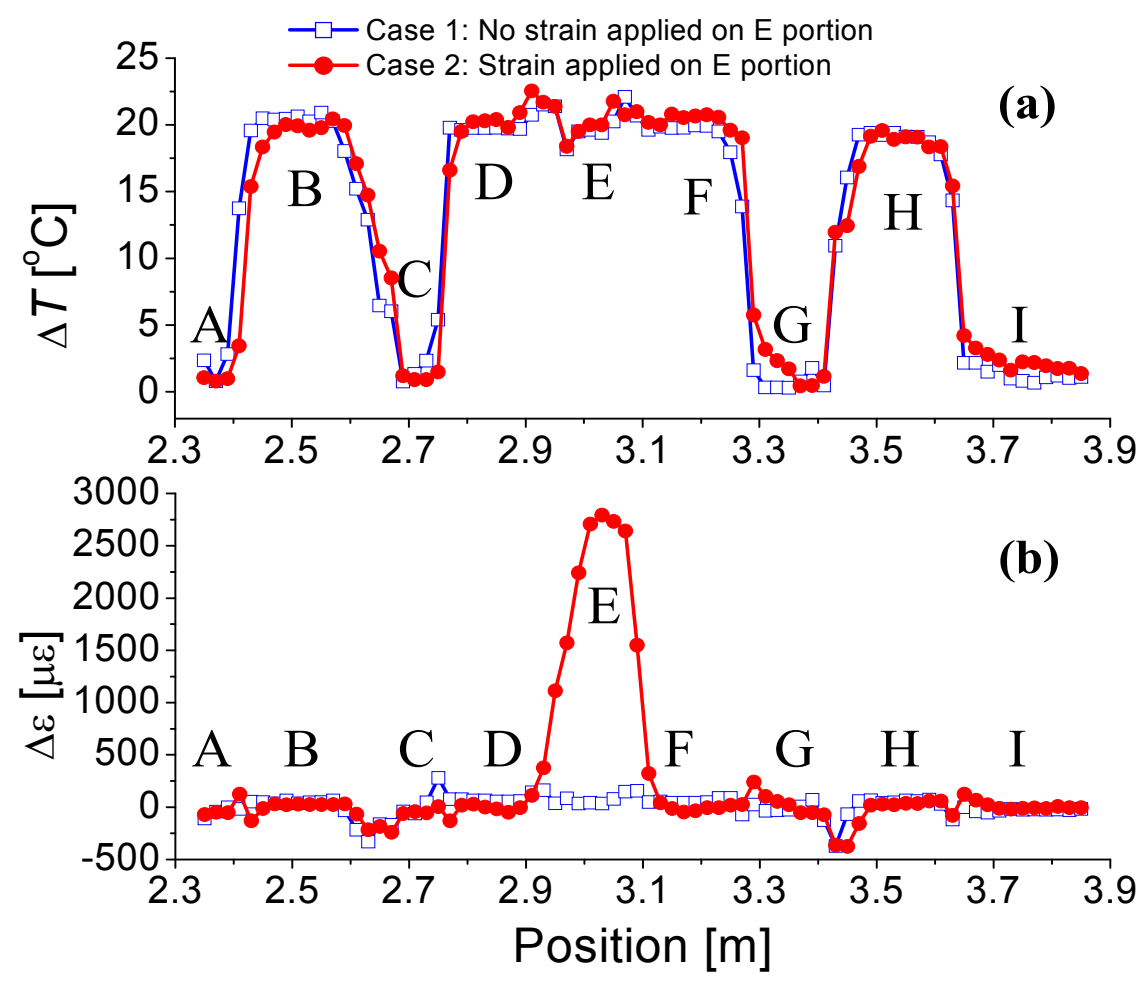

Fig. 2: Distributed discriminative measurement of temperature (a) and strain (b) [39]. Squares: B, D, E, F and H sections are heated. Dots: Section E is additionally strained. 
crack detection in concrete [21], multi-points dynamic strain measurement in a building model [22], and aircraft health monitoring using a business jet-plane [23].

\section{Brillouin dynamic grating and applications}

We demonstrated an optically controllable grating in an optical fiber, "Dynamic Grating," utilizing gain saturation phenomenon in an erbium-doped optical fiber [24] in 2003, and also realized distributed strain sensing [25]. In 2007, we newly demonstrated Brillouin Dynamic Grating (BDG) generation in a polarization maintaining (PM) optical fiber $[26,27]$ in our laboratory in the University of Tokyo, collaborating with Chung-Ang University, Korea. The longitudinal acoustic wave generated by two lightwaves counter-propagating as one polarization mode is found to make Bragg reflection for the third lightwave launched as the other polarization mode [26,27]. We also demonstrated distributed measurement of the Bragg reflection spectra, or the BDG spectra, along the PM fiber both with correlation domain
[28] and time domain [29] schemes. Recently, application and characterization of the BDG have been studied, including distributed temperature [30] and birefringence [31] measurements, observation of its intrinsic spectra [32,33], controllable optical delay lines [34-37], and so on.

We have characterized the dependence of the BDG spectrum on temperature and strain [38], and proposed a scheme for distributed discriminative measurement of strain and temperature with a high accuracy by using a PM fiber [39]. Figure 1 shows the system configuration for the distributed discriminative measurement with the correlation domain technique [39]. In Fig. 2, the measured results are shown, in which the high spatial resolution of about $10 \mathrm{~cm}$ has been achieved with BOCDA technique [39]. Recently, the measurement accuracy [40] and range [41] have been improved with one-laser-based generation and detection of the BDG. The distributed discriminative measurements have also been performed in time domain schemes [42,43]. The discriminative measurement of strain and

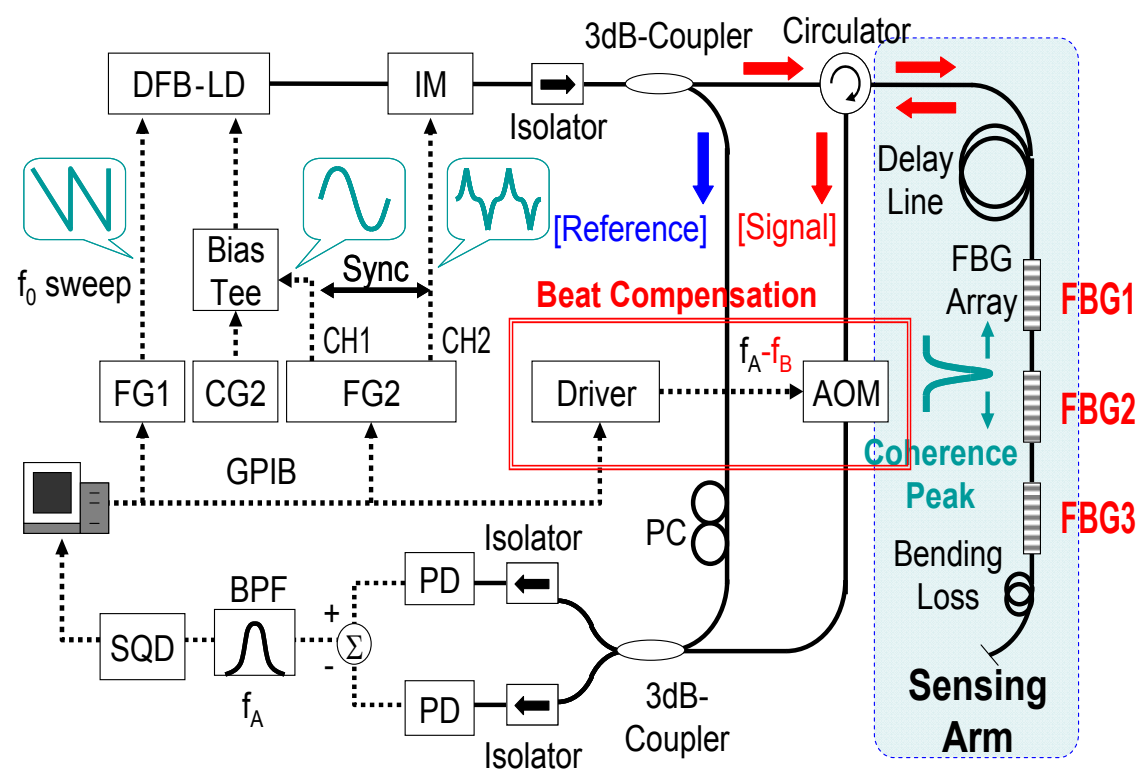

Fig. 3: System for multiplexing long-length FBG distributed sensors [49]. With the correlation domain technique, Bragg wavelength distribution inside the FBGs can be measured. 

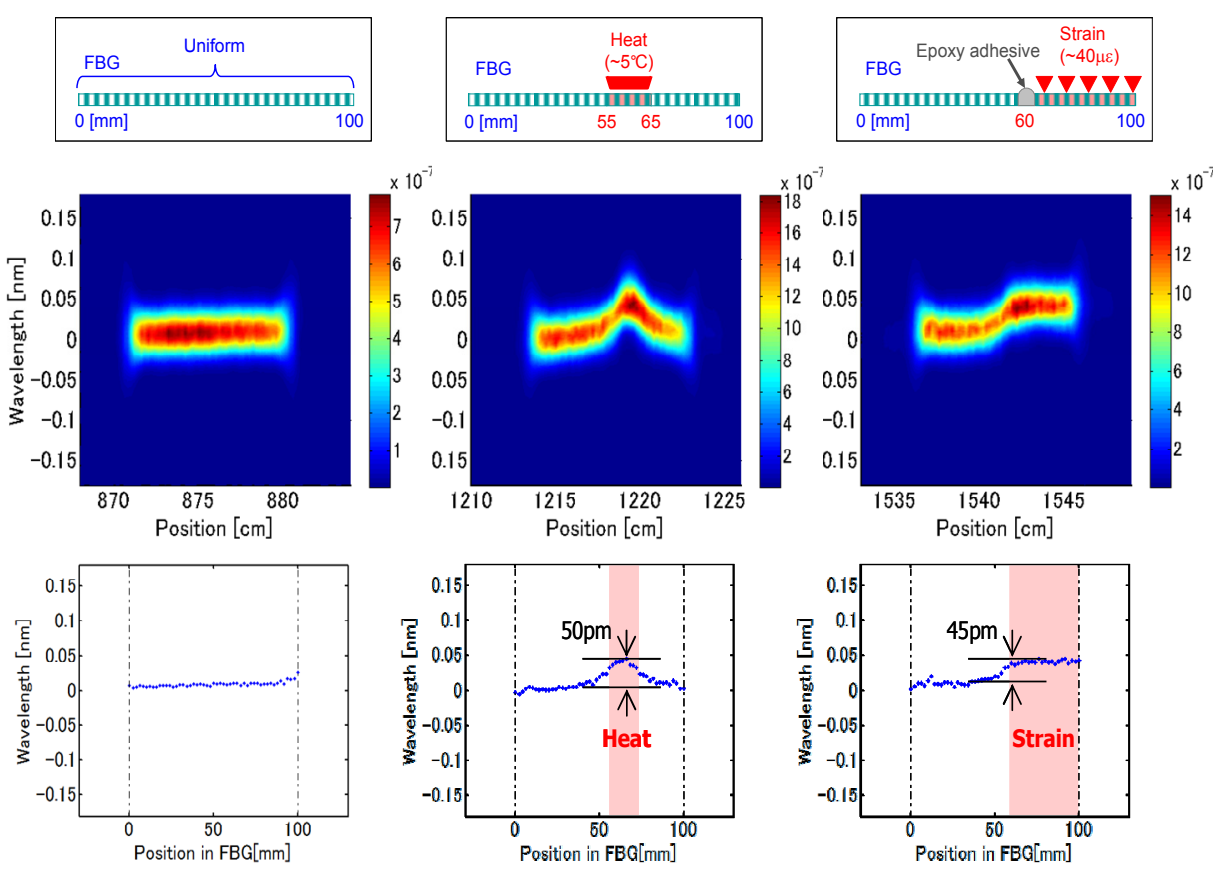

Fig. 4. Bragg wavelength distribution measured inside the three multiplexed long-length FBG sensors [49]. FBG1; no perturbation, FBG2; heat at center, FBG3; strain at right half.

temperature is quite important for practical applications of the fiber optic nerve systems. Recently, combination of the Brillouin frequency shift and the Brillouin intensity has been used for this purpose [44]. To achieve $10 \mathrm{~cm}$ order spatial resolution, the BOCDA technique has been adopted in the system [44].

Another interesting scheme realized with the BDG is high spatial resolution distributed Brillouin gain spectrum measurement with time domain technique $[45,46]$, and $1 \mathrm{~cm}$ resolution has already been demonstrated [46].

\section{Multiplexed long-length FBG sensors}

Fiber Bragg grating based multiplexed strain sensing has been widely studied. We have proposed a scheme, in which the FBGs with the same Bragg wavelength can be multiplexed by using the correlation domain technique. By a scheme introduced to enhance the measurement speed, dynamic strain measurement has been demonstrated with $10 \mathrm{kHz}$ sampling rate. Recently, we have also developed a system, in which the Bragg wavelength distribution inside a long-length FBG can be measured by the correlation domain technique [47]. Measurement rate has been improved to be 1 $\mathrm{kHz}$ for one point [48]. Even when changing the point, $100 \mathrm{~Hz}$ has already been achieved [48]. Figure 3 shows the system, in which the longlength FBG sensors are multiplexed [49]. Figure 4 shows the spectra and the Bragg wavelength distributions measured along three $100 \mathrm{~mm}$ length FBGs [49].

\section{Conclusions}

Brillouin scattering based distributed sensing schemes have been reviewed. Time domain techniques have recently been improved in spatial resolution. In correlation domain techniques, superior functions have already been demonstrated, such as $\mathrm{mm}$ order resolution and random accessibility. Brillouin dynamic grating is the promising phenomenon, creating new functions, such as distributed discriminative measurement of strain and temperature. Quasi-distributed sensing scheme with multiplexed long-length distributed FBG sensors has also been shown, utilizing a correlation domain technique. 